\title{
Course of sepsis in rats with thyroid dysfunction
}

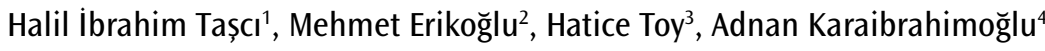

ABSTRACT

Cite this paper as:

Taşcı Hi, Erikoğlu M, Toy H,

Karaibrahimoğlu A. Course of sepsis in rats with thyroid dysfunction. Turk J Surg 2017; 33: $175-179$

This study was presented at the $50^{\text {th }}$ Congress of the European Society for Surgical Research,

10-13 June 2015, Liverpool, UK.

'Department of General Surgery, Reyhanli Public Hospital, Hatay, Turkey

2Department of General Surgery, Necmettin Erbakan University Meram School of Medicine, Konya, Turkey

${ }^{3}$ Department of Pathology, Necmettin Erbakan University Meram School of Medicine, Konya, Turkey

${ }^{4}$ Department of Medical

Statistics, Necmettin Erbakan University Meram School of

Medicine, Konya, Turkey

\section{Address for Correspondence} Halil İbrahim Taşa e-mail: okcu1@mynet.com

Received: 20.05 .2016 Accepted: 10.08.2016

\section{CCopyright 2017} by Turkish Surgical Association

Available online at www.turkjsurg.com
Objective: Numerous studies show the relationship between sepsis and thyroid hormones. Virtually all these studies investigate changes in post-sepsis thyroid hormones and the relationship between these changes and the progression of the disease. Our aim in this study was to investigate the progression of sepsis in rats with thyroid dysfunction. Material and Methods: The study involved four groups, each containing seven female Wistar albino rats: Group 1: Sham, Group 2: Control (Sepsis), Group 3: Hyperthyroidism-Sepsis, and Group 4: Hypothyroidism-Sepsis. Group 1 only received laparotomy. Group 2 only had sepsis. Sepsis was induced in Group 3 and Group 4 following formation of hyperthyroidism and hypothyroidism, respectively. After 24 hours, relaparotomy and thoracotomy were performed, and tissue and blood samples were drawn.

Results: Dysfunctions seen in the liver, lungs, and kidneys during sepsis and other findings of sepsis were milder in the hyperthyroidism group in comparison to both the control and hypothyroidism groups.

Conclusion: The results of Simon's grade, histopathological organ damage, and laboratory parameters revealed that the progression of sepsis was milder in the hyperthyroid group than in the hypothyroid and euthyroid groups. The progression in the hypothyroid group was the most severe. Therefore, the results of the study raise the question of whether immediate treatment in cases of hypothyroidism and slow return of thyroid function to normal levels in cases of hyperthyroidism are adequate treatment approaches in patients who may develop sepsis or septic shock." To determine the answer to this question, more detailed studies are required with a higher number of subjects.

Keywords: Hyperthyroidism, hypothyroidism, organ dysfunction, sepsis

\section{INTRODUCTION}

Critical diseases cause hormonal changes that may vary during the acute and chronic phases of the disease (1). Changes are also seen during disease along the hypothalamic-pituitary-thyroid (HPT) axis. These changes are typically related to a decrease in the total 3, 5, 3'-L-triiodothyronine (T3) level. The term nonthyroidal illness syndrome (NTIS) is used to describe the changes in thyroid-related hormone levels during acute or chronic illness (2). Decrease in T3 is the most common finding in NTIS. The only finding may be decreased T3 levels in diseases with relatively mild progression. As the severity of disease increases, decreases in 3, 5, 3'-L-tetraiodothyronine (T4) and thyroid stimulant hormone (TSH) levels along with T3 and elevation of reverse T3 level are seen (3). Some studies have linked this change in thyroid hormones during sepsis and septic shock with poor prognosis (4). Studies demonstrating the relationship between relative hypothyroidism and poor prognosis have focused on thyroid hormone treatment during sepsis.

Numerous studies in the literature show a relationship between sepsis and thyroid hormones. Virtually all these studies investigated changes in post-sepsis thyroid hormones and the relationship between these changes and the progression of the disease. However, in this study, we aimed to investigate the progression of sepsis in rats with previous conditions of hyperthyroidism or hypothyroidism.

\section{MATERIAL AND METHODS}

This study was approved by Necmettin Erbakan University KONUDAM Experimental Medicine Research and Study Center's local ethics board for animal trials on 11.14.2014 (decree 2014-056); all institutional and national guidelines for the care and use of laboratory animals were followed. The study was conducted at Necmettin Erbakan University KONUDAM Experimental Medicine Research and Study Center. All procedures related to animals were performed in adherence to the Strasbourg Contract (ETS 123 and ETS 170) and Directive 2010/63/Eu of the European Parliament and of the Council of 22 September 2010 on the protection of animals used for scientific purposes.

Twenty-eight female Wistar albino rats weighing 260 to 320 grams were used in the study. Four groups, each containing seven animals, were formed. Group 1 was designated as the sham group, while Group 2 was the control sepsis group, Group 3 was the hyperthyroidism + sepsis group, and Group 4 was the hypothyroidism + sepsis group. All animals were fed with standard feed and water for 15 days. Before the experiment, the rats were housed in wire cages at a temperature of $22^{\circ} \mathrm{C}$ and $50 \%$ to $60 \%$ humidity with 12 hours night, 12 hours day circadian rhythms. A maximum of five rats were housed in a single cage. Solid foods were removed from the rats' diet 12 hours before surgical procedures, and water was removed 2 hours prior to surgery. The rats in 
Group 3 were administered a daily dose of $250 \mu \mathrm{g} / \mathrm{kg}$ L-thyroxine (Sigma) dissolved in $0.1 \mathrm{~N} \mathrm{NaOH}$ and diluted with normal saline intraperitoneally (i.p.) for 15 days; thus, hyperthyroidism was established (5). The animals in Group 4 were i.p. injected with $0.5 \mathrm{ml}$ 6-n-propyl-2-thiouracil (Sigma) dissolved in isotonic solution for 15 days at a daily dose of $10 \mathrm{mg} / \mathrm{kg}$ (6). Before the surgical procedures, all animals were anesthetized with $50 \mathrm{mg} / \mathrm{kg}$ ketamine $\mathrm{HCl}$ and $15 \mathrm{mg} / \mathrm{kg}$ xylazine subcutaneously. Following anesthesia, at the end of the $15^{\text {th }}$ day, all the rats underwent mini-laparotomy operations with a midline incision. The rats in the sham group did not undergo further procedures; their abdomens were closed in two layers with 4/0 silk after their cecae were isolated. Experimental sepsis formed in the other groups (groups 2, 3, and 4) in line with the cecal ligation and perforation (CLP) model (7) (Figure 1). To achieve this, the cecum was isolated; after the visible colon was patted and filled with feces, it was ligated with $3 / 0$ silk below the iliocecal valve; the cecum was perforated with an 18-gauge branule needle, and the abdomen was continuously closed in two layers with $4 / 0$ silk. After the procedure, a $2 \mathrm{mg} / \mathrm{mL}$ dose of paracetamol tablets was mixed in drinking water to provide analgesia. At the end of 24 hours, anesthesia was performed in the same way; all rats underwent relaparatomy and thoracotomy, and intraabdominal inflammation was graded according to Simon's method (8) (Table 1). Intracardiac blood was drawn for biochemical and hormonal analyses. Tissue samples from the liver, lungs, and kidneys were acquired for pathological diagnoses. The animals were euthanized under anesthesia by exsanguination.

Renal function tests (urea, creatinine), hepatic function tests (AST, ALT), C-reactive protein (CRP), thyroid tests (fT3, fT4, TSH), and blood gas parameters $\left(\mathrm{pH}, \mathrm{pCO}_{2}, \mathrm{pO}_{2}\right.$, lactate, $\left.\mathrm{SaO}_{2}, \mathrm{HCO}_{3}\right)$ were analyzed in the drawn blood. The following were evaluated under a light microscope by a pathologist who had no knowledge of the groups: in liver specimens: inflammation, congestion, Kupffer cell hyperplasia, and hydropic degeneration; in lung specimens: inflammation, congestion, and atelectasis; in kidney specimens: inflammation, congestion, and tubular cell degeneration.

\section{Statistical Analysis}

The statistical analysis of the collected data was conducted using the Statistical Package for the Social Sciences (IBM Corp.; Armonk, NY, USA) 20.0 software package. When parametric conditions were met for intergroup comparisons, one-way analysis of variance (ANOVA) was used, while Kruskal-Wallis variance analysis was implemented for other cases. For binary comparisons, the post-hoc Tukey test was preferred. For the comparison of two groups, Student's t-test or the Mann-Whitney U test was used. To determine whether categorical variables were related, chi-square analysis was used; to pinpoint the relationships between numeric variables, Pearson's and Spearman's correlation tests were used. $P<0.05$ was regarded to be statistically significant for all analyses.

\section{RESULTS}

One rat from the hypothyroidism group died 18 hours after implementation of the sepsis model in the course of the study. This rat underwent relaparotomy and thoracotomy immediately after mortality. Following the grading of intraabdominal sepsis according to Simon's method, tissue samples were taken. Because no blood samples could be drawn from this rat, the laboratory parameters were evaluated over six rats in this group.

24 hours after completion of the CLP procedure, it was seen that reluctance to feed, and somnolence became more prominent, especially in the hypothyroidism and control sepsis groups.

With respect to Simon's grade, the hyperthyroidism group had a more moderate outlook than the control sepsis group. The results of the hypothyroidism group, however, were poorer than those of the other three groups (Table 2, Figure 2). Among the clinical parameters, Simon's grade had negative correlations with fT3 ( $p=0.029)$ and fT4 ( $p=0.004)$; however, no correlations were seen with regard to TSH level $(p=0.268)$.

fT3 $(p=0.002)$, fT4 $(p=0.016)$, and TSH $(p<0.001)$ levels were found to be significantly different in all groups (Table 3 ). The fT3 level was the highest in the sham group and was the lowest in the hypothyroidism group. Binary group comparisons of the fT4 levels demonstrated that the hormone level was significantly lower only in the hypothyroidism group than in the sham group $(p=0.013)$. TSH levels were found to be highest in the hypothyroidism group.

Binary group comparisons of the neutrophil counts revealed that the sham group was not statistically significantly different from the hyperthyroidism group $(p=0.121)$; in contrast, the results in hypothyroid group were the poorest (Table 3). While $\mathrm{pH}$ levels were statistically lower in the hypothyroidism and control sepsis groups than in the sham group $(p=0.049$ and $p=0.035$, respectively), the results of the sham group and the hyperthyroidism group were not significantly different ( $\mathrm{p}=0.953) . \mathrm{pCO}_{2}$ levels were found to be higher in the control sepsis group $(p=0.027)$ and the hypothyroidism group $(p=0.017)$ than in the sham group, whereas the results of the hyperthyroidism group $(p=0.978)$ and the sham group were not statistically significantly different (Table 3). AST, ALT, and CRP were also significantly higher in the hyperthyroidism and hypothyroidism groups than in the sham group $(p<0.005)$.

When the organ damage was histopathologically evaluated, liver, lung, and kidney damage were most severe in the hypothyroidism group, followed by the control sepsis group. The results of the hyperthyroidism group were better than both groups (Table 4, Figure 3-5). Also, there was a negative correlation between hepatic and renal failure and fT3 level $(p<0.005)$.

\section{DISCUSSION}

Sepsis is described as a systemic disease condition brought about by microbial invasion of normally sterile areas of the body. Sepsis conditions accompanied by hypoperfusion and at least one organ dysfunction are called severe sepsis; if vasopressor need or hypotension, which are seen during adequate fluid resuscitation, is added to severe sepsis, it is called septic shock (9). The results of our study showed that the disease was less severe in the hy-

Table 1. Classification of peritoneal inflammation according to Simon's method

Grade 0 No inflammation

Grade 1 Well-walled-off abscess, no free peritoneal fluid

Grade 2 Walled-off abscess, small amount of free peritoneal fluid, and patchy areas of cecal wall necrosis

Grade 3 Poor localization of the inflammatory process, moderate amount of free peritoneal fluid, and gangrenous cecum

Grade 4 No walling off of the cecum, large amount of free hemorrhagic fluid in the peritoneal cavity, and extensive gangrene and hemorrhagic fluid in the cecal wall 
perthyroidism group than in both the hypothyroidism and the control sepsis groups. Moreover, not only laboratory parameters, but also histopathological symptoms referring to liver, lung, and kidney damage were better in the hyperthyroidism group than in either the control sepsis or hypothyroidism groups.

Among the inflammatory molecules secreted by the host and used for the diagnosis of infection, the presence of bacteremia, and the progress and mortality of disease are TNF-a, IL 6, CRP, and procalcitonin, which is recently being used more frequently (10). Thyroid hormones are a point of interest in this regard. Decrease in T3 is the most frequently seen finding; it may be the only find-

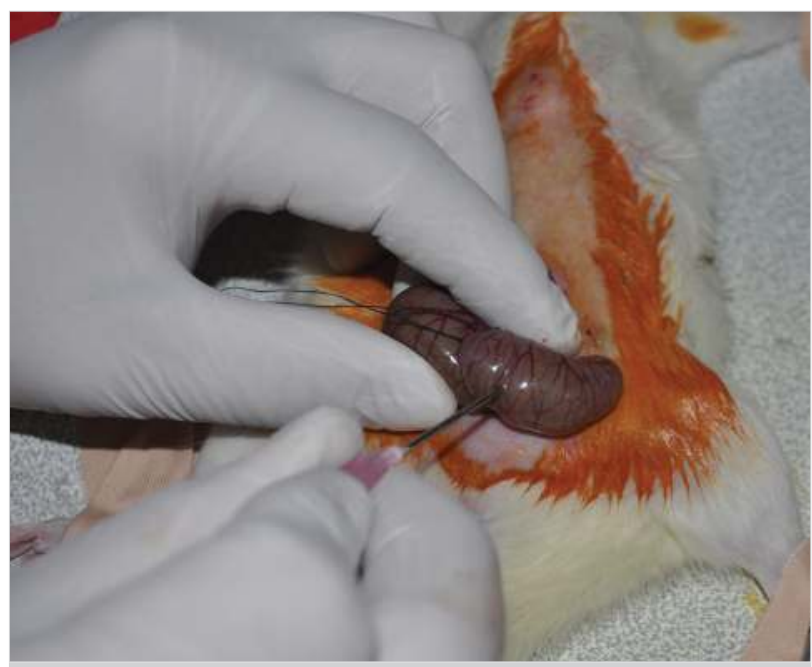

Figure 1. Cecal ligation and perforation

Table 2. The distribution of Simon's grades according to the groups

\begin{tabular}{|lcccc|} 
Simon's grade & $\begin{array}{c}\text { Group 1 } \\
(n=7)\end{array}$ & $\begin{array}{c}\text { Group 2 } \\
(n=7)\end{array}$ & $\begin{array}{c}\text { Group 3 } \\
(n=7)\end{array}$ & $\begin{array}{c}\text { Group 4 } \\
(n=7)\end{array}$ \\
\hline Grade 0 & 7 & - & - & - \\
\hline Grade 1 & - & - & - & - \\
\hline Grade 2 & - & 2 & 4 & 2 \\
\hline Grade 3 & - & 4 & 3 & 2 \\
\hline Grade 4 & - & 1 & - & 3 \\
\hline
\end{tabular}

ing in T3 in diseases with milder progression. As the severity of the disease increases, T4 and TSH levels decrease along with T3, and an increase in reverse T3 level is observed (3). In our study, we concluded that fT3 and fT4 levels were lowest in the hypothyroidism group, followed by the control sepsis, hyperthyroidism, and sham groups. In contrast, the TSH level was significantly higher in the hypothyroidism group. This situation was related to the antithyroid treatment administered before the CLP procedure.

Recent studies have argued that low thyroid hormone levels are signifiers of poor outcomes in sepsis (11). The results of a review study conducted by Angelousi et al. (4), which examined nine studies on the subject, revealed that six of nine studies demonstrated significantly lower total or free T3 and T4 levels in deteriorating septic patients than in other patients. In another study by Sharma et al. (12) investigating the relationship between thyroid hormone dysfunction and CRP levels during sepsis in newborns, the authors demonstrated a close relation-

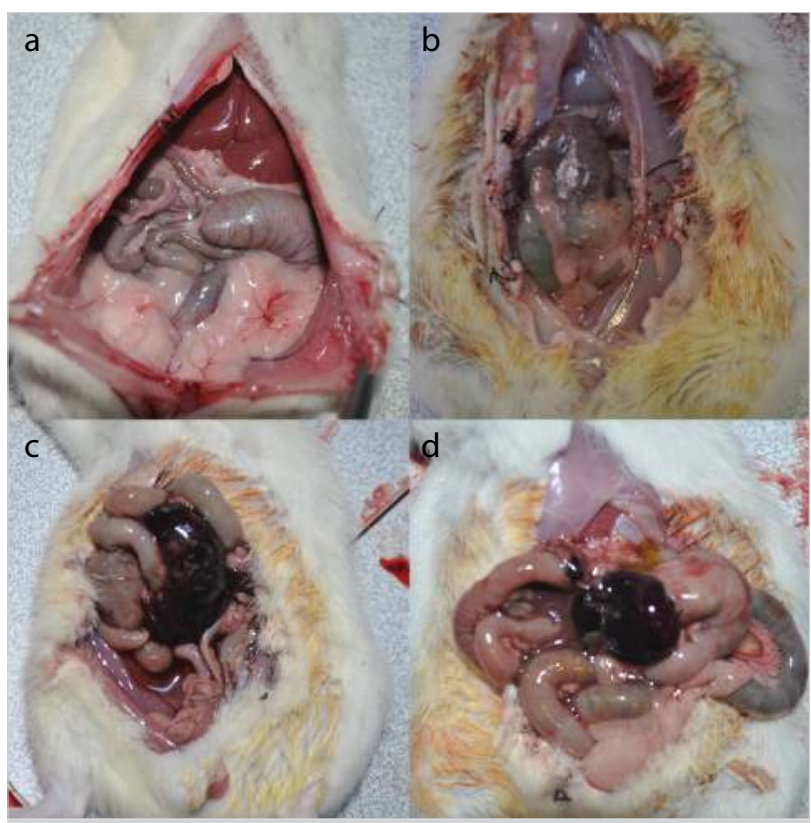

Figure 2. a-d. Simon's grades. Grade 0 (an animal from the sham group) (a). Grade 2 (an animal from the hyperthyroidism group) (b). Grade 3 (an animal from the control sepsis group) (c). Grade 4 (an animal from the hypothyroidism group) (d)

Table 3. Blood sample results

\begin{tabular}{|c|c|c|c|c|c|}
\hline Blood parameter & Group $1(n=7)$ & Group $2(n=7)$ & Group $3(n=7)$ & Group $4(n=6)$ & $\mathbf{p}$ \\
\hline fT3 $(\mathrm{pg} / \mathrm{mL})$ & $2.27 \pm 0.38$ & $1.52 \pm 0.35$ & $2.21 \pm 0.54$ & $1.49 \pm 0.38$ & 0.002 \\
\hline TSH ( $\mu \mathrm{IU} / \mathrm{mL})$ & $0.47 \pm 0.18$ & $0.40 \pm 0.29$ & $0.33 \pm 0.25$ & $2.53 \pm 0.52$ & $<0.001$ \\
\hline Neutrophils & $2.7 \pm 0.77$ & $7.77 \pm 3.43$ & $5.80 \pm 1.56$ & $8.50 \pm 3.30$ & 0.001 \\
\hline $\mathrm{pH}$ & $7.33 \pm 0.7$ & $7.21 \pm 0.09$ & $7.31 \pm 0.80$ & $7.21 \pm 0.62$ & 0.013 \\
\hline $\mathrm{pCO}_{2}(\mathrm{mmHg})$ & $44.24 \pm 10.60$ & $58.82 \pm 5.51$ & $46.15 \pm 9.62$ & $60.43 \pm 9.33$ & 0.004 \\
\hline AST (u/L) & $152.57 \pm 61.61$ & $287.71 \pm 97.36$ & $314.14 \pm 116.00$ & $358.33 \pm 87.59$ & 0.03 \\
\hline $\mathrm{ALT}(\mathrm{u} / \mathrm{L})$ & $65.57 \pm 22.26$ & $163.28 \pm 62.94$ & $230.14 \pm 86.40$ & $237.16 \pm 43.27$ & $<0.001$ \\
\hline Urea (mg/dL) & $31.77 \pm 7.31$ & $88.24 \pm 35.41$ & $119.92 \pm 34.68$ & $122.86 \pm 44.65$ & $<0.001$ \\
\hline
\end{tabular}




\begin{tabular}{|c|c|c|c|c|c|c|}
\hline Tissue samples & Histopathological findings & & Grup $1(n=7)$ & Grup2 $(n=7)$ & Grup $3(n=7)$ & Grup $4(n=7)$ \\
\hline \multirow[t]{16}{*}{ Liver } & \multirow[t]{4}{*}{ Inflammatory cell infiltration } & 0 & 7 & 2 & 7 & 1 \\
\hline & & $1+$ & - & 4 & - & 3 \\
\hline & & $2+$ & - & 1 & - & 3 \\
\hline & & $3+$ & - & - & - & - \\
\hline & \multirow[t]{4}{*}{ Congestion } & 0 & 2 & - & 5 & - \\
\hline & & $1+$ & 5 & 1 & 2 & - \\
\hline & & $2+$ & - & 6 & - & 5 \\
\hline & & $3+$ & - & - & - & 2 \\
\hline & \multirow{4}{*}{ Kupffer cell hyperplasia } & 0 & 7 & 7 & 7 & 7 \\
\hline & & $1+$ & - & - & - & - \\
\hline & & $2+$ & - & - & - & - \\
\hline & & $3+$ & - & - & - & - \\
\hline & \multirow[t]{4}{*}{ Hydropic degeneration } & 0 & 7 & 7 & 7 & 7 \\
\hline & & $1+$ & - & - & - & - \\
\hline & & $2+$ & - & - & - & - \\
\hline & & $3+$ & - & - & - & - \\
\hline \multirow[t]{12}{*}{ Lung } & \multirow[t]{4}{*}{ Inflammatory cell infiltration } & 0 & 7 & - & - & - \\
\hline & & $1+$ & - & - & - & - \\
\hline & & $2+$ & - & 4 & 4 & 4 \\
\hline & & $3+$ & - & 3 & 3 & 3 \\
\hline & \multirow[t]{4}{*}{ Congestion } & 0 & 3 & - & - & - \\
\hline & & $1+$ & 4 & - & - & - \\
\hline & & $2+$ & - & 5 & 6 & 2 \\
\hline & & $3+$ & - & 2 & 1 & 5 \\
\hline & \multirow[t]{4}{*}{ Atelectasis } & 0 & 2 & - & - & - \\
\hline & & $1+$ & 5 & - & 1 & - \\
\hline & & $2+$ & - & 4 & 2 & 3 \\
\hline & & $3+$ & - & 3 & 4 & 4 \\
\hline \multirow[t]{12}{*}{ Kidney } & \multirow[t]{4}{*}{ Inflammatory cell infiltration } & 0 & 7 & 5 & 7 & 4 \\
\hline & & $1+$ & - & 2 & - & 3 \\
\hline & & $2+$ & - & - & - & - \\
\hline & & $3+$ & - & - & - & - \\
\hline & \multirow[t]{4}{*}{ Congestion } & 0 & 4 & - & 4 & - \\
\hline & & $1+$ & 3 & 2 & 3 & - \\
\hline & & $2+$ & - & 5 & - & 6 \\
\hline & & $3+$ & - & - & - & 1 \\
\hline & \multirow[t]{4}{*}{ Tubular cell degeneration } & 0 & 7 & - & 2 & - \\
\hline & & $1+$ & - & 3 & 5 & 6 \\
\hline & & $2+$ & - & 4 & - & 1 \\
\hline & & $3+$ & - & - & - & - \\
\hline
\end{tabular}

ship between low fT3 level and high CRP level. Furthermore, Wang et al. (13) proved that fT3 was related to intensive care mortality in a prospective study that compared intensive care mortality and thyroid function. Numerous studies have been conducted, in addition to those mentioned above, on the relationship between thyroid hormones and the progression of sepsis. These studies reveal conflicting results regarding whether changes in thyroid hormones are related to the progression of sepsis, whether these changes are signifiers of mortality, and which thyroid hormones are better signifiers. The main cause of these inconsistencies is that almost all these studies were observational ones conducted with adult or pediatric intensive care patients. Therefore, it is not known whether the patients in the studies had previous conditions of underlying thyroid disease. Most drugs used in intensive care (such as dopaminergic agents) may lead to thyroid dysfunction; some studies did not exclude patients receiving these drugs. Most of the patients had comorbid diseases that could affect thyroid functions; these patients were not excluded in some studies. In contrast, in our study, we addressed the problems that limit the dependability of some previous studies; also, the relationship between thyroid hormone levels and the progression of sepsis was evaluated in a

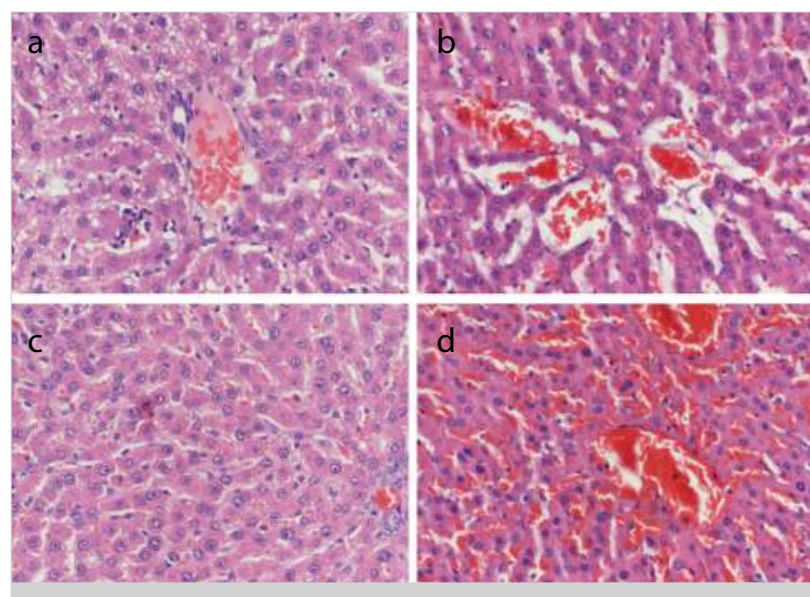

Figure 3. a-d. Hepatic tissue $(H \& E \times 200)$. Sample from the sham group (histopathologically normal outlook) (a). Sample from the control sepsis group (pronounced congestion and sporadic inflammatory cell infiltration) (b). Sample from the hyperthyroidism group (histopathologically normal outlook) (c). Sample from the hypothyroidism group (advanced congestion and inflammatory cell infiltration) (d) 


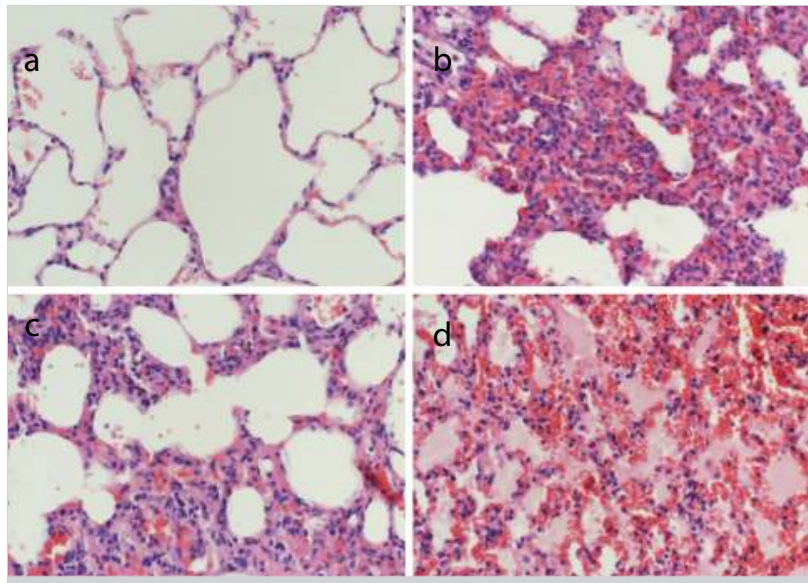

Figure 4. a-d. Lung tissue (H\&E-200). Sample from the sham group (histopathologically normal outlook) (a). Sample from the control sepsis group (extensive inflammatory cell infiltration, congestion, and atelectasis areas) (b). Sample from the hyperthyroidism group (inflammatory cell infiltration, mild atelectasis, and congestion areas) (c). Sample from the hypothyroidism group (advanced congestion, pronounced atelectasis areas, and inflammatory cell infiltration accompanied by plasma cell infiltration in the alveoli) (d)

negative correlations between the clinical phase of the disease and fT3 ( $p=0.029$ ) and fT4 ( $p=0.004$ ) levels; however, no correlations were seen with regard to TSH level $(p=0.268)$. There was a negative correlation between hepatic and renal failure and fT3 level $(p<0.005)$. In light of these data, it was concluded that fT3 is a more valuable parameter in demonstrating damage, especially histopathological damage.

\section{CONCLUSION}

Simon's grade, histopathological organ damage, and laboratory parameter results revealed that progression of sepsis was less severe in the hyperthyroid group than in the hypothyroid and euthyroid groups. The progression of sepsis was most severe in the hypothyroid group. Therefore, the results of this study raise the question of whether immediate treatment in cases of hypothyroidism and slow return of thyroid function to normal levels in cases of hyperthyroidism are adequate treatment approaches in patients who may develop sepsis or septic shock." To determine the answer to this question, more detailed studies with a higher number of subjects are necessary.

Ethics Committee Approval: Ethics committee approval was received for this study from the ethics committee of Necmettin Erbakan University KONUDAM Experimental Medicine Research and Study Center's (date: 11.14.2014/decision no: 2014-056).

Informed Consent: Not required in this study.

Peer-review: Externally peer-reviewed.

Author Contributions: Concept - H.I.T., M.E.; Design - H.I.T., M.E.; Supervision - H.I.T., M.E.; Materials - H.I.T., A.K.; Data Collection and/or Processing - H.I.T., A.K.; Analysis and/or Interpretation - H.I.T., A.K.; Literature Search - H.I.T.; Writing Manuscript - H.I.T., M.E.; Critical Reviews - H.I.T., M.E., A.K.

Conflict of Interest: No conflict of interest was declared by the authors.

Financial Disclosure: The authors declared that this study has received no financial support.

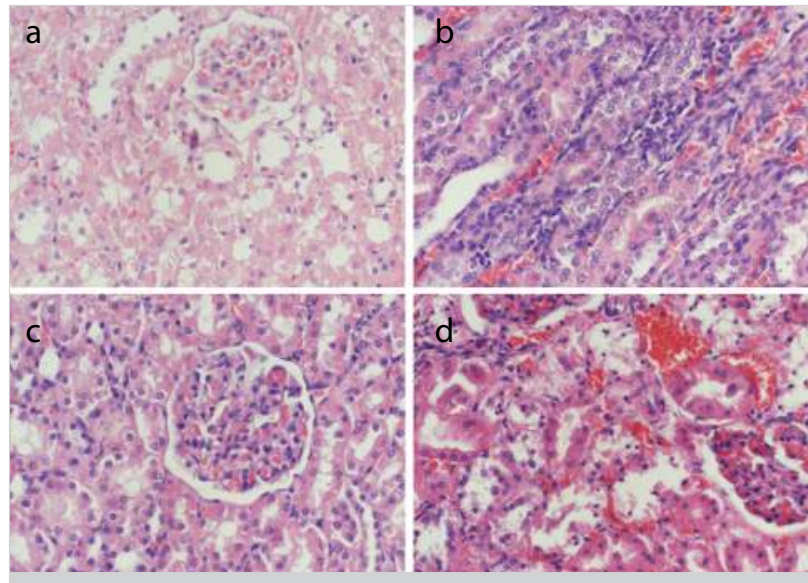

Figure 5. a-d. Kidney tissue $(H \& E \times 200)$. Sample from the sham group (histopathologically normal outlook) (a). Sample from the control sepsis group (sporadic inflammatory cell infiltration, congestion, and pronounced tubule cell degeneration) (b). Sample from the hyperthyroidism group (histopathologically normal outlook) (c). Sample from the hypothyroidism group (pronounced congestion and tubule cell degeneration) (d)

\section{REFERENCES}

1. Van den Berghe G. Dynamic neuroendocrine responses to critical illness. Front Neuroendocrinol 2002; 23: 370-391. [CrossRef]

2. Warner $M H$, Becket GJ. Mechanisms behind the non-thyroidal illness syndrome: an update. J Endocrinol 2010; 205: 1-13. [CrossRef]

3. Mclver B, Gorman CA. Euthyroid sick syndrome: an overview. Thyroid 1997; 7: 125-132. [CrossRef]

4. Angelousi AG, Karageoropoulos DE, Kapaskelis AM, Falagas ME. Association between thyroid function tests at baseline and the outcome of patients with sepsis or septic shock: a systematic review. Eur J Endocrinol 2011; 164: 147-155. [CrossRef]

5. Moğulkoç R, Baltacı AK. Effect of intraperitoneal melatonin supplementation on releases of thyroid hormones and testosterone in hyperthyroid rat. Genel Tıp Derg 2002; 12: 129-132.

6. Kükner A, Öner J, Öner H, Çolakoğlu N, Özoğul C, Ozan E. Light and electron microscopic observations in the rat thyroid following experimental hypothyroidism. Firat Medical J 2001; 2: 202208.

7. Otero-Anton E, Gonzalez-Quintela A, Lopez Soto A, Lopez-Ben S, Llovo J, Perez LF. Cecal ligation and puncture as amodel of sepsis in the rat: influence of the puncture size on mortality, bacteremia, endotoxemia and tumor necrosis factor alpha levels. Eur Surg Res 2001; 33: 77-79. [CrossRef]

8. Simon R, Wetzel N, Winsey K, Levenson SM, Demetriou AA Supplemental dietary tyrosine in sepsis and acute hemorrhagic shock. Arch Surg 1987; 122: 78-81. [CrossRef]

9. Lever A, Mackenzie I. Sepsis: definition, epidemiology, and diagnosis. BMJ 2007; 335: 879-883. [CrossRef]

10. Chan YL, Tseng CP, Tsay PK, Chang SS, Chiu TF, Chen JC. Procalcitonin as a marker of bacterial infection in the emergency department: an observational study. Crit Care 2004; 8: 12-20. [CrossRef]

11. Van den Berghe G, Zegher F, Bouillon R. Clinical review 95: acute and prolonged critical illness as different neuroendocrine paradigms. J. Clin Endocrinol Metab 1998; 83: 1827-1834. [CrossRef]

12. Sharma S, Dabla PK, Kumar S, Dublis S. Thyroid hormone dysfunction and CRP levels in neonates with sepsis. J Endocrinol Metab 2013; 3: 62-66. [CrossRef]

13. Wang F, Pan W, Wang H, Wang S, Pan S, Ge J. Relationship between thyroid function and ICU mortality: a prospective observation study. Crit Care 2012; 16: R11. [CrossRef] 